\title{
Isolation and propagation of Trypanosoma brucei gambiense from sleeping sickness patients in south Sudan
}

\author{
Naomi W.N. Maina a,b,1, Michael Oberle ${ }^{b}$, Charles Otieno $^{a}$, Christina Kunz $^{b}$, \\ Pascal Maeser ${ }^{c}$, Joseph M. Ndung' ${ }^{d}$, Reto Brun ${ }^{b, *}$
}

\author{
a Trypanosomiasis Research Centre (TRC) of KARI, P.O. Box 362, Kikuyu, Kenya \\ b Department of Medical Parasitology and Infection Biology, Swiss Tropical Institute, P.O. Box, CH-4002 Basel, Switzerland \\ c University of Bern, Institute of Cell Biology, Baltzerstrasse 4, CH-3012 Bern, Switzerland \\ ${ }^{\mathrm{d}}$ Foundation for Innovative New Diagnostics (FIND), Geneva, Switzerland
}

Received 27 June 2006; received in revised form 1 November 2006; accepted 1 November 2006 Available online 1 February 2007

\author{
KEYWORDS \\ Sleeping sickness; \\ Trypanosoma brucei \\ gambiense; \\ Cryopreservation; \\ Isolation; \\ Mastomys natalensis; \\ SCID mice; \\ Sudan
}

\begin{abstract}
Summary This study aimed at isolating Trypanosoma brucei gambiense from human African trypanosomiasis (HAT) patients from south Sudan. Fifty HAT patients identified during active screening surveys were recruited, most of whom (49/50) were in second-stage disease. Blood and cerebrospinal fluid samples collected from the patients were cryopreserved using Triladyl ${ }^{\circledR}$ as the cryomedium. The samples were stored at $-150^{\circ} \mathrm{C}$ in liquid nitrogen vapour in a dry shipper. Eighteen patient stabilates could be propagated in immunosuppressed Mastomys natalensis and/or SCID mice. Parasitaemia was highest in SCID mice. Further subpassages in $M$. natalensis increased the virulence of the trypanosomes and all 18 isolates recovered from $M$. natalensis or SCID mice became infective to other immunosuppressed mouse breeds. A comparison of immunosuppressed M. natalensis and Swiss White, C57/BL and BALB/C mice demonstrated that all rodent breeds were susceptible after the second subpassage and developed a parasitaemia $>10^{6} / \mathrm{ml}$ by Day 5 post infection. The highest parasitaemias were achieved in $\mathrm{C} 57 / \mathrm{BL}$ and BALB/c mice. These results indicate that propagation of $T . b$. gambiense isolates after initial isolation in immunosuppressed $M$. natalensis or SCID mice can be done in a range of immunosuppressed rodents.

(c) 2006 Royal Society of Tropical Medicine and Hygiene. Published by Elsevier Ltd. All rights reserved.
\end{abstract}

\section{Introduction}

\footnotetext{
* Corresponding author. Tel.: +41 61284 8231; fax: +41612848101.

E-mail address: reto.brun@unibas.ch (R. Brun).

1 Present address: Jomo Kenyatta University of Agricultural Technology, P.O. Box 62000, Nairobi, Kenya.
}

African sleeping sickness (or human African trypanosomiasis (HAT)) is endemic in 36 countries in sub-Saharan Africa. It is estimated that 60 million people are at risk of infection, that 500000 people are already infected and approximately 


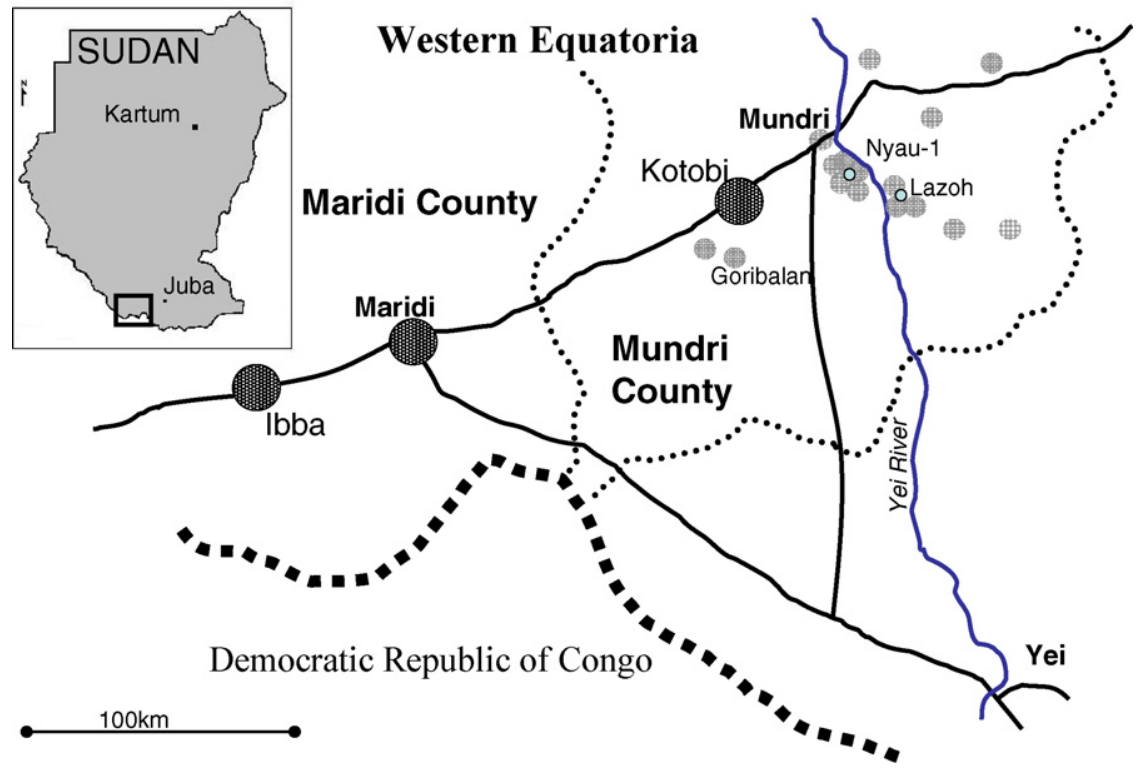

Figure 1 Study site in Western Equatoria in south Sudan.

50000 die every year (WHO, 2004). The disease is caused by Trypanosoma brucei rhodesiense and T. b. gambiense. The former causes an acute disease in Eastern Africa, whilst $T$. b. gambiense generally causes a chronic disease in Western and Central Africa. In Sudan, HAT due to T. $b$. gambiense is highly endemic, with an estimated $1-2$ million people at risk of infection and thousands of people infected.

Control of the disease relies mainly on chemotherapy. The drugs used in Sudan are pentamidine for first-stage disease and melarsoprol and eflornithine for second-stage disease. Melarsoprol is normally the first-line drug for treatment of second-stage disease. In recent years, high rates of melarsoprol treatment failure $(>20 \%)$ have been reported in T. $b$. gambiense-endemic areas in Uganda, Angola and Sudan (Burri and Keiser, 2001; Legros et al., 1999; Moore and Richer, 2001). Additionally, a high fatality rate following melarsoprol treatment has been noted. This has led to a change of treatment policy and since September 2001 eflornithine became the first-line drug for second-stage disease in Ibba Hospital (Médicins Sans Frontières, 2002).

The role of resistant trypanosomes in treatment failures is not well understood, mainly because of a lack of $T$. b. gambiense isolates available from various endemic countries. One reason for this is the difficulty of isolating this trypanosome species and propagating it in laboratory rodents (Brun et al., 2001). The purpose of this study was to isolate T. b. gambiense from patients in Ibba Hospital and to evaluate a new cryopreservation medium, Triladyl ${ }^{\circledR}$ (Maina et al., 2006), under field conditions. Additionally, the capacity of different mouse breeds to develop a parasitaemia sufficient for laboratory investigations was studied.

\section{Materials and methods}

\subsection{Isolation of trypanosomes from patients}

Active screening of HAT patients was carried out by Médecins Sans Frontières France (MSF-F) in Mundri and Maridi coun- ties, Western Equatoria, south Sudan (Figure 1). Diagnosis was done serologically (Magnus et al., 1978) or parasitologically (trypanosomes detected either in lymph node aspirate, venous blood or cerebrospinal fluid (CSF)). The number of white cells in CSF was determined and used for classification of the stage of disease (WHO, 1998). Patients who had been treated for HAT less than 12 months previously were considered relapses or treatment failures.

In the hospital, patients were informed of the objectives and protocol of the study. To be included in the study, the patients (or their guardians) had to give their consent (written/thumb print). The WHO and the Ministry of Health of Sudan People's Liberation Army (SPLA) approved the study protocol.

\subsubsection{Cryopreservation of patient stabilates}

Samples of $2-3 \mathrm{ml}$ of venous blood were collected from patients into heparinised vacutainer tubes. Samples of $2 \mathrm{ml}$ of CSF were also collected from two self-reporting patients attending the hospital. Trypanosomes in the samples were concentrated by centrifugation at $10000 \mathrm{rpm}$ for $10 \mathrm{~min}$ and the pellet was re-suspended in Triladyl ${ }^{\circledR}$ at a 1:1 ratio for blood and 1:10 for CSF. Aliquots of $500 \mu$ l were then transferred into Nunc ${ }^{\circledR}$ ampoules (Nunc, Roskilde, Denmark). Four to five stabilates were prepared per patient and stored in a liquid nitrogen dry shipper in the vapour phase at $-150^{\circ} \mathrm{C}$. Dry shippers containing the stabilates were then transferred from the field sites to the Trypanosomiasis Research Centre (TRC) in Kenya where primary propagation in rodents was carried out.

\subsection{Propagation of Trypanosoma brucei gambiense in laboratory rodents}

\subsubsection{Laboratory animals}

Mastomys natalensis and Swiss White mice were bred at TRC. C57/BL and BALB/c mice, respectively, were purchased from the International Livestock Research Institute (ILRI) 
and the Kenya Medical Research Institute (KEMRI), both in Nairobi, Kenya. Severe combined immunodeficient (SCID), $\mathrm{C} 3 \mathrm{H}$ and FVB mice were purchased from the Charles River Laboratories, Inc. (Munich, Germany). NMRI mice were purchased from RCC Ltd. (Itingen, Switzerland). The animals were housed under conventional conditions and fed on commercial pellets and water ad libitum.

All animals (except SCID mice) were immunosuppressed by administration of cyclophosphamide at $300 \mathrm{mg} / \mathrm{kg}$ body weight applied intraperitoneally 1 day prior to infection and were treated every 10 days with $200 \mathrm{mg} / \mathrm{kg}$.

\subsubsection{Primary propagation in Mastomys natalensis and SCID mice}

The presence and viability of trypanosomes in the stabilates was determined by inoculating one stabilate into one SCID mouse and two immunosuppressed $M$. natalensis. The stabilates were thawed rapidly in a water-bath at $37^{\circ} \mathrm{C}$ and immediately injected intraperitoneally into the rodents. Parasitaemia was monitored every other day for 60 days by examination of tail blood using the haematocrit centrifugation technique (HCT) and/or the method of Herbert and Lumsden (1976). Parasitaemic animals were euthanised using $\mathrm{CO}_{2}$ and blood was collected by cardiac puncture. The infected blood from the first passage was cryopreserved in Triladyl ${ }^{\circledR}$ and stored in liquid nitrogen. Successful stabilates were given an isolation code different from the original patient stabilate.

Rodents that remained aparasitaemic for 60 days after inoculation with cryopreserved material were euthanised and blood was collected by cardiac puncture. If no parasites could be detected using the HCT method, another two $M$. natalensis were inoculated with another duplicate stabilate.

\subsubsection{Susceptibility of various strains of mice to Trypanosoma brucei gambiense isolates}

The growth pattern of five randomly selected isolates was determined in $\mathrm{C} 57 / \mathrm{BL}, \mathrm{BALB} / \mathrm{C}$, Swiss White, NMRI, $\mathrm{C} 3 \mathrm{H}$ and FVB mice as well as in $M$. natalensis. Prior to that, parasites from the primary propagation were passaged twice in $M$. natalensis. Trypanosomes were harvested from a donor $M$. natalensis and inoculated into groups of four animals ( $4 \times 10^{5}$ trypanosomes/mouse) of the different mouse breeds. Thawing of the stabilates, inoculation and parasitaemia determination were performed as described earlier.

\subsection{Genotypic characterisation}

Trypanosomes (passage 1) were purified using an anion exchange column (Lanham and Godfrey, 1970) and DNA was extracted using the Puregene DNA extraction kit (Gentra Systems, Minneapolis, MN, USA) as previously described by Matovu et al. (2001a).

Amplification of the serum resistance-associated (SRA) gene and the T. b. gambiense-specific glycoprotein (TgsGP) gene was done as previously described by Radwanska et al. (2002a, 2002b). However, for both genes a nested PCR was performed. For SRA, the two sets of primers used were: SRA-out-s (5'-CCTGATAAAACAAGTATCGGCAGCAA-3') and SRA-out-as (5'-CGGTGACCAATTCATCTGCTGCTGTT-3'); and SRA-inner-s (5'-ATA GTG ACA TGC GTA CTC AAC GC-3') and SRA-inner-as (5'-AAT GTG TTC GAG TAC TTC GGT CAC GCT-3'). For the TgsGP gene, the primer pairs were: TbsGP-outer-s (5'-GCGTATGCGATACCGCAGTAA-3') and TbsGP-outer-as (5'-GCTTCAACCGCCGCTGCTTCTA-3'); and TbsGP-s (5'-GCTGCTGTGTTCGGAGAGC-3') and TbsGP-as (5' GCCATCGTGCTTGCCGCTC-3').

For control PCRs, the actin gene was amplified using primers act-s (5'-CCGAGTCACACAACGT-3') and act-as (5'-CCACCTGCATAACATTG-3'). The amplifications were performed in $50 \mu$ l of reaction mixture containing PCR buffer (Qiagen, Basel, Switzerland), $200 \mathrm{mM}$ of the four dNTPs, $1 \mu \mathrm{M}$ of each primer and Taq polymerase in a PTC 200 Peltier thermocycler (MJ Research, Waltham, MA, USA) under the following conditions: initial denaturation at $94^{\circ} \mathrm{C}$ for $3 \mathrm{~min}$, 30 cycles of $94^{\circ} \mathrm{C}$ for $45 \mathrm{~s}, 62^{\circ} \mathrm{C}\left(55^{\circ} \mathrm{C}\right.$ for actin) for $45 \mathrm{~s}$ and $72{ }^{\circ} \mathrm{C}$ for $120 \mathrm{~s}$, followed by a final extension for $10 \mathrm{~min}$ and rapid cooling at $4{ }^{\circ} \mathrm{C}$.

Eight microlitres of the PCR product were analysed by electrophoresis in a $1.25 \%$ agarose gel. The gels were stained with ethidium bromide $(0.2 \mu \mathrm{g} / \mathrm{ml})$ and visualised under UV light.

\subsubsection{Sequencing of TgsGP}

For sequencing, two independent PCR reactions were pooled, purified using QIAquick columns (Qiagen) and sequenced with the Big Dye Terminator kit (Applied Biosystems, Foster City, CA, USA). The products were run at the Computational and Molecular Population Genetic Laboratories (CMGP, Zoology Department, University of Bern) on an $A B I$ sequencer.

\section{Results}

\subsection{Patients}

Blood samples were collected from 50 patients from Mundri (46), Maridi (2), Yambio (1) and Juba (1) counties (Figure 1). The mean age of the patients was 24 years (range $0.25-62$ years) with $>90 \%$ below 40 years of age. Most patients $(49 / 50)$ were in the second stage of the disease, which was mainly based on a white cell count $>5$ cells $/ \mu$ l rather than the presence of trypanosomes in the CSF. Seven of the patients had previously been treated (7-12 months before): four with eflornithine (at $400 \mathrm{mg} / \mathrm{kg} /$ day for 14 days) and three with pentamidine ( $4 \mathrm{mg} / \mathrm{kg} /$ day for 7 days).

Forty-two patients were parasite positive in the blood and eight were aparasitaemic but card agglutination test for trypanosomiasis (CATT) positive at a 1:4 dilution. Samples of CSF were only collected from two patients attending the hospital for passive screening. For all other patients, a lumbar puncture had already been done in the field during active screening and for ethical reasons a second lumbar puncture was not performed in the hospital.

\subsection{Primary propagation of stabilates from patients}

Of the 50 blood samples inoculated into $M$. natalensis and SCID mice, 18 caused patent infections. The two CSF sam- 
Table 1 Trypanosoma brucei gambiense isolates from Mundri county that could be propagated in laboratory rodents

\begin{tabular}{|c|c|c|c|c|c|c|c|c|c|c|c|}
\hline \multirow{3}{*}{$\begin{array}{l}\text { Patient } \\
\text { stabilate }\end{array}$} & \multirow[t]{3}{*}{ Isolate no. } & \multicolumn{3}{|c|}{ Patient data } & \multicolumn{3}{|c|}{ Proof of infection and staging ${ }^{a}$} & \multicolumn{4}{|c|}{ Primary propagation in rodents ${ }^{b}$} \\
\hline & & \multirow{2}{*}{$\begin{array}{l}\text { Bomas } \\
\text { (village) }\end{array}$} & \multirow{2}{*}{$\begin{array}{l}\text { Age } \\
\text { (years) }\end{array}$} & \multirow[t]{2}{*}{ Sex } & \multirow{2}{*}{$\begin{array}{l}\text { Blood/ } \\
\text { lymph }\end{array}$} & \multirow[t]{2}{*}{ CSF } & \multirow{2}{*}{$\begin{array}{l}\text { CSF } \\
(\text { cells } / \mu l)\end{array}$} & \multicolumn{2}{|l|}{ SCID mice } & \multicolumn{2}{|c|}{ M. natalensis } \\
\hline & & & & & & & & $\begin{array}{l}\text { Pre-patent } \\
\text { period }\end{array}$ & $\begin{array}{l}\text { Parasitaemia at freezing } \\
\text { (days post infection) }\end{array}$ & $\begin{array}{l}\text { Pre-patent } \\
\text { period }\end{array}$ & $\begin{array}{l}\text { Parasitaemia at freezing } \\
\text { (days post infection) }\end{array}$ \\
\hline 104008 & Mundri 01 & Midi & 19 & $\mathrm{~F}$ & + & - & 8 & 7 & $1.6 \times 10^{7}(7)$ & 12 & $+++(14)$ \\
\hline 103037 & Mundri 02 & Amandi & 12 & $\mathrm{~F}$ & + & - & 49 & 7 & $1.6 \times 10^{7}(7)$ & 8 & $+++(10)$ \\
\hline K03028 & Mundri 03 & Nyau 1 & 25 & M & + & - & 6 & 11 & $1.6 \times 10^{7}(12)$ & 4 & $+++(6)$ \\
\hline K03048 & Mundri 04 & Buangyi & 20 & M & + & - & 58 & 4 & $1.6 \times 10^{7}(6)$ & 4 & $2.5 \times 10^{4}(5)$ \\
\hline K03051 & Mundri 05 & Nyau 1 & 23 & M & ND & - & 50 & 7 & $8 \times 10^{6}(14)$ & 23 & $++(26)$ \\
\hline K03030 & Mundri 06 & Goribalan & 21 & $\mathrm{~F}$ & + & - & 4 & 11 & $1 \times 10^{6}(21)$ & 9 & $++(11)$ \\
\hline K03042 & Mundri 07 & Nyau 1 & 20 & M & + & - & 10 & 14 & $++(14)$ & 10 & $++(12)$ \\
\hline 104019 & Mundri 08 & Lazoh & 10 & $M$ & + & - & 17 & 13 & $+(13)$ & 21 & $3.2 \times 10^{7}(25)$ \\
\hline K03043 & Mundri 09 & Wiri Lui & 6 & M & + & - & 9 & 5 & $3.2 \times 10^{7}(9)$ & - & - \\
\hline 104015 & Mundri 10 & Singo & 12 & $M$ & + & - & 104 & 11 & $1.6 \times 10^{7}(11)$ & - & - \\
\hline K03045 & Mundri 11 & Nyau 1 & 12 & M & ND & - & 8 & 17 & $1.0 \times 10^{6}(22)$ & - & - \\
\hline 104022 & Mundri 12 & Lazoh & 9 & $\mathrm{~F}$ & + & - & 8 & 16 & $2.5 \times 10^{4}(24)$ & - & - \\
\hline K03010 & Mundri 13 & Mundri & 27 & M & + & - & 61 & - & - & 3.5 & $8 \times 10^{6}(5)$ \\
\hline 104021 & Mundri 14 & Gingo & 23 & $\mathrm{~F}$ & + & - & 45 & - & - & 18 & $2 \times 10^{6}(21)$ \\
\hline 103030 & Mundri 15 & Lazoh & 12 & $\mathrm{~F}$ & + & - & 8 & - & - & 6 & $2.5 \times 10^{4}(7)$ \\
\hline K03044 & Mundri 16 & Nyau 1 & 12 & $\mathrm{~F}$ & + & - & 20 & - & - & 11 & $+++(13)$ \\
\hline K03032 & Mundri 17 & Singirigwa & 22 & $\mathrm{~F}$ & + & + & 783 & - & - & 17 & $+++(18)$ \\
\hline K03054 & Mundri 18 & Moto & 25 & $M$ & + & - & 22 & - & - & 11 & $+(13)$ \\
\hline
\end{tabular}

CSF: cerebrospinal fluid; ND: not done.

a Trypanosomes were detected by examination of blood using haematocrit centrifugation technique (HCT) method and/or lymph node aspirate and by examination of CSF. Staging of disease was done by examination of CSF for the presence of trypanosomes and by white blood cell count in CSF ( $>5$ cells $/ \mu \mathrm{l}=\mathrm{second}$-stage disease).

b Primary propagation was done in immunosuppressed Mastomys natalensis and SCID mice. Parasitaemia in rodents was estimated by the method of Herbert and Lumsden (1976) or, for lower parasitaemia, by the HCT method. 
ples did not lead to patent parasitaemia in the rodents. Patient stabilates that could be successfully propagated in the rodents were designated with the name 'Mundri' (the name of the county where all the patients came from) and a consecutive number; furthermore, they were termed isolates (Table 1). A slightly higher recovery was obtained in M. natalensis $(14 / 18)$ compared with SCID mice $(12 / 18)$. Only $8 / 18$ stabilates could be propagated in both rodents. The stabilates had a similar pre-patent period in SCID mice (10.25 \pm 4.26 days) and $M$. natalensis (11.25 \pm 6.3 days). However, in $M$. natalensis only $3 / 14$ stabilates developed a parasitaemia of $\geq 10^{6} / \mathrm{ml}$, whereas in the SCID mice $9 / 12$ stabilates reached that level of parasitaemia. Details of these 18 isolates are shown in Table 1.

\subsubsection{Determination of the suitability of Swiss White mice for patient stabilates}

Only five (103030, I03037, K03048, K03045 and K03051) of the 18 patient stabilates that could be propagated in either immunosuppressed $M$. natalensis and/or in SCID mice caused patent infection in immunosuppressed Swiss White mice. The susceptibility of Swiss White mice was much lower compared with that of $M$. natalensis. The parasitaemias in Swiss White mice were generally low (apart from K03048) and could only be detected by the HCT method. For one stabilate (K03051), parasitaemia was sporadic and inconsistent.

\subsubsection{Susceptibility of mice to secondary propagation of Trypanosoma brucei gambiense}

All 18 isolates (Mundri 1 to 18 , first passage) inoculated into immunosuppressed Swiss White mice caused patent infection, with a pre-patent period of 4 days. Most of the isolates $(11 / 18)$ caused low and inconsistent parasitaemias $\left(<10^{4} / \mathrm{ml}\right)$, but the remaining isolates (Mundri 02, 03, 04, 06, 08,14 and 17) grew to higher parasitaemia with levels $>10^{6}$ trypanosomes/ml (data not shown).

The growth patterns of five randomly selected isolates (passage 4) were compared in immunosuppressed $\mathrm{C} 57 / \mathrm{BL}$, $\mathrm{BALB} / \mathrm{C}$ and Swiss White mice and M. natalensis (Table 2). The parasites were infective for all rodents used, with a similar pre-patent period of 3 days and a parasitaemia $>10^{6}$ trypanosomes/ml by Day 5 . There was variation in the growth pattern between the isolates and between rodent breeds. For all five isolates, the highest parasitaemia $\left(>5 \times 10^{7}\right.$ trypanosomes $\left./ \mathrm{ml}\right)$ was noted in $\mathrm{C} 57 / \mathrm{BL}$ mice. Overall, C57/BL and BALB/C mice sustained higher parasitaemias $\left(>10^{6} / \mathrm{ml}\right)$ for several days compared with Swiss White mice or $M$. natalensis. In all isolates, the parasitaemias dropped to undetectable levels by Day 16 post infection. Very few animals (4/80) died during the 60-day observation period. In NMRI, $\mathrm{C} 3 \mathrm{H}$ and FVB mice, parasitaemia was always very low and far below the parasitaemia in $\mathrm{C} 57 / \mathrm{BL}$ and BALB/C mice (data not shown).

\subsection{Genotypic characterisation}

Trypanosoma brucei subspecies-specific genotyping was performed by PCR using the SRA gene as a marker for T. $b$. rhodesiense (Radwanska et al., 2002a) and the TgsGP gene for T. b. gambiense (Berberof et al., 2001). The marker genes were amplified from genomic DNA by nested PCR

Table 2 Comparison of the growth characteristics of Trypanosoma brucei gambiense in different rodents. Animals were immunosuppressed with cyclophosphamide $300 \mathrm{mg} / \mathrm{kg}$ prior to infection. Each animal was inoculated with $4 \times 10^{4}$ trypanosomes (passage 3) by the intraperitoneal route. Parasitaemia was monitored every other day by examination of the tail blood for 20 days $^{\mathrm{a}}$

\begin{tabular}{|c|c|c|c|}
\hline Isolate no. (patient stabilate) & Rodent breed & $\begin{array}{l}\text { Period with parasitaemia } \\
>10^{6} / \mathrm{ml} \text { (mean duration) (days) }\end{array}$ & $\begin{array}{l}\text { Highest parasitaemia } \\
\text { (days post infection) }\end{array}$ \\
\hline \multirow{5}{*}{ Mundri 02 (I03037) } & C57/BL & $4-16(12.5)$ & $2.5 \times 10^{8}(7)$ \\
\hline & $\mathrm{BALB} / \mathrm{C}$ & $4-14(4.25)$ & $1.6 \times 10^{7}(8.5)$ \\
\hline & Swiss White & $5-7(2)$ & $0.8 \times 10^{7}(6)$ \\
\hline & Mastomys natalensis & $6-8(2)$ & $3.2 \times 10^{6}(7)$ \\
\hline & $\mathrm{C} 57 / \mathrm{BL}$ & $5-9(4.66)$ & $5.0 \times 10^{7}(7.66)$ \\
\hline \multirow{3}{*}{ Mundri 05 (K03051) } & $\mathrm{BALB} / \mathrm{c}$ & - & - \\
\hline & Swiss White & $5-16(4.5)$ & $1.3 \times 10^{7}(8.25)$ \\
\hline & M. natalensis & $6-7(1.5)$ & $4.6 \times 10^{6}(6)$ \\
\hline \multirow{4}{*}{ Mundri 09 (K03043) } & $\mathrm{C} 57 / \mathrm{BL}$ & $5-8(3.75)$ & $7.4 \times 10^{7}(6.75)$ \\
\hline & $\mathrm{BALB} / \mathrm{c}$ & $5-9(4.75)$ & $6.4 \times 10^{7}(7.25)$ \\
\hline & Swiss White & $5-7(3)$ & $1.4 \times 10^{6}(6.5)$ \\
\hline & M. natalensis & $5-7(3)$ & $0.8 \times 10^{7}(6)$ \\
\hline \multirow{4}{*}{ Mundri 13 (K03010) } & $\mathrm{C} 57 / \mathrm{BL}$ & $5-14(6.25)$ & $1.5 \times 10^{8}(7.5)$ \\
\hline & $\mathrm{BALB} / \mathrm{c}$ & $5-14(6.26)$ & $8.9 \times 10^{7}(7.5)$ \\
\hline & Swiss White & $5-7(1.75)$ & $3.2 \times 10^{6}(6.25)$ \\
\hline & M. natalensis & $5-7(2.5)$ & $1.1 \times 10^{7}(5)$ \\
\hline \multirow{4}{*}{ Mundri 17 (K03032) } & $\mathrm{C} 57 / \mathrm{BL}$ & $5-18(13)$ & $5.0 \times 10^{8}(14)$ \\
\hline & $\mathrm{BALB} / \mathrm{c}$ & $6-9(3.33)$ & $5.0 \times 10^{7}(7.33)$ \\
\hline & Swiss White & $6-7(2)$ & $5.0 \times 10^{6}(6.5)$ \\
\hline & M. natalensis & $5-7(3)$ & $6.4 \times 10^{7}(7)$ \\
\hline
\end{tabular}

\footnotetext{
${ }^{a}$ Data shown are the mean of four animals.
} 


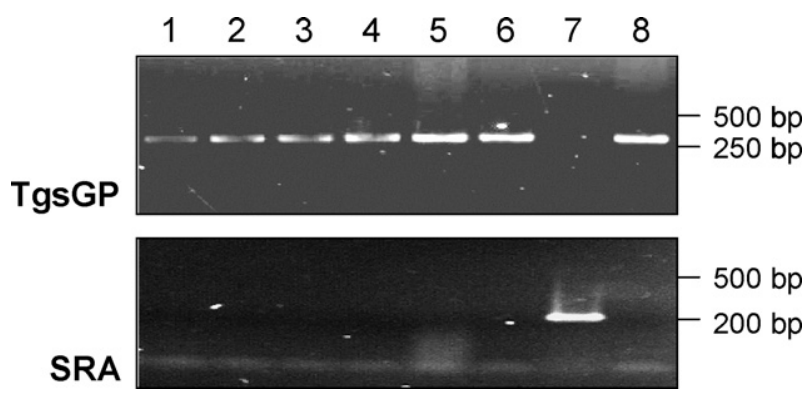

Figure 2 Genotypic characterisation: amplification of the Trypanosoma brucei gambiense-specific glycoprotein gene (TgsGP, top) and the serum resistance-associated gene (SRA, bottom) in trypanosomes isolated from human African trypanosomiasis patients. All 18 stabilates gave the same result, therefore only 6 of them are shown here (lanes 1-6). Trypanosoma $b$. rhodesiense STIB 704 (lane 7) and T. b. gambiense STIB 754 (lane 8) were used as reference.

and were analysed on agarose gels (Figure 2). All isolates tested were positive for TgSGP and negative for SRA. To verify that the correct gene was amplified, PCR products were directly sequenced. Apart from a few silent mutations, the obtained sequence (GenBank accession no. DQ224158) was identical to that published for TgsGP (GenBank accession no. AJ277951), confirming that the isolated trypanosomes were indeed $T$. $b$. gambiense.

\section{Discussion}

This is the first report of successful isolation of T. b. gambiense from patients in south Sudan. Eighteen viable isolates could be cryopreserved under field conditions using the new cryomedium Triladyl ${ }^{\circledR}$ (Maina et al., 2006). In previous studies, a low recovery rate or complete loss of $T$. $b$. gambiense populations following cryopreservation in medium containing 10\% glycerol have been reported (Brun et al., 2001; Matovu et al., 2001b). The recovery rate achieved of $43 \%$ is very satisfactory and may be attributed to the superior cryopreservation medium Triladyl ${ }^{\circledR}$.

Viability of the isolates in rodents depended on the degree of parasitaemia in the patients. Parasitaemia in patients was always low and only detectable by HCT; in eight patients parasites were not detectable at all although the patients were CATT positive. Earlier reports also noted the poor infectivity of $T$. b. gambiense for laboratory rodents, leading to a low isolation success (Aerts et al., 1992; Dukes et al., 1989).

In the current study, we used the African rodent M. natalensis (immunosuppressed) for primary propagation of the patient stabilates on a comparative basis with SCID mice, which have no functional T- and B-lymphocytes (Bosma et al., 1983). Mastomys natalensis was reported to be a better model for T. b. gambiense than rats and mice (Mehlitz, 1978) and has been extensively used for propagation studies (Aerts et al., 1992; Dukes et al., 1989; Gibson et al., 1978; Matovu et al., 2001b; Zillmann and Mehlitz, 1979). Inoue et al. (1998) first described the high susceptibility of SCID mice to $T$. b. gambiense isolates, a finding that was confirmed in our study. However, the use of SCID mice is restricted by the high cost of the mice as well as availability. On the other hand, breeding colonies of M. natalensis are already in place at institutes in East Africa.

Normal inbred or outbred laboratory mice have generally not been found to be susceptible to $T$. $b$. gambiense isolates, although they can easily be infected with T. $b$. rhodesiense trypanosomes. However, we found that following immunosuppression different breeds of laboratory mice could be infected with the second or third passage of our Mundri T. b. gambiense isolates. Even stabilates prepared directly from patients were infective for immunosuppressed Swiss White mice, although with a lower success rate $(5 / 18)$ compared with $M$. natalensis $(14 / 18)$ or SCID mice $(12 / 18)$. However, after a second passage, all 18 isolates were infective for immunosuppressed Swiss White mice and 7 of them developed a parasitaemia $>10^{6} / \mathrm{ml}$. In other mouse strains (C57/BL, BALB/C, NMRI, C3H and FVB), the parasitaemia was very low, except for the three isolates $\mathrm{K} 03028, \mathrm{~K} 03048$

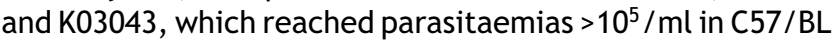
and BALB/C by Day 14 post infection. Passaging the isolates at least three times increased their virulence, resulting in parasitaemias $>10^{7} / \mathrm{ml}$.

In conclusion, we recommend that infected blood samples from $T$. $b$. gambiense patients should be cryopreserved in Triladyl ${ }^{\circledR}$ medium. Primary propagation and subsequent subpassage should be done in immunosuppressed $M$. natalensis or in SCID mice. Further subpassages can then be done in immunosuppressed laboratory mice (e.g. C57/BL or BALB/C). Available mouse breeds should be screened following immunosuppression with cyclophosphamide at $300 \mathrm{mg} / \mathrm{kg}$ prior to infection and repeated once per week at $200 \mathrm{mg} / \mathrm{kg}$. This propagation scheme is efficient as well as economical. However, it must be kept in mind that repeated passage through rodents can lead to parasite strain selection, thus the results obtained must not be interpreted as representing the true strain diversity in the human population. The proposed scheme will allow the characterisation of $T$. $b$. gambiense from various hot spots of Central Africa and therefore facilitate the monitoring of drug-resistant trypanosomes. These new T. b. gambiense isolates from Sudan are a unique source for further scientific investigations. Their characterisation, especially for drug sensitivity, will help to elucidate the cause of the high melarsoprol treatment failure rates reported from the area.

\section{Conflicts of interest statement}

The authors have no conflicts of interest concerning the work reported in this paper.

\section{Authors' contributions}

NWNM, JMN and RB designed the study outline; NWNM and MO carried out the fieldwork; NWNM, CO and CK carried out the animal experiments; PM supervised the genotypic characterisation; NWNM and RB drafted the manuscript. All authors read and approved the final manuscript. NWNM and $\mathrm{RB}$ are guarantors of the paper.

\section{Acknowledgements}

We would like to thank the Eastern Africa Network for Trypanosomosis (EANETT) and the Swiss Agency for Devel- 
opment and Co-operation (SDC) for financial support. We are grateful to Médecins Sans Frontières France (MSF-F) for allowing us to work under their sleeping sickness control programme in south Sudan and for their efficient logistic planning of the field trip. The technical assistance provided by Guy Riccio (STI), Rashid Farah (TRC) and Peter Waweru (TRC) is gratefully acknowledged.

\section{References}

Aerts, D., Truc, P., Pencheir, L., Claes, Y., Le Ray, D., 1992. A kit for in vitro isolation of trypanosomes in the field: first trial with sleeping sickness patients in the Congo Republic. Trans. R. Soc. Trop. Med. Hyg. 86, 394-395.

Berberof, M., Perez-Morga, D., Pays, E., 2001. A receptorlike flagellar pocket glycoprotein specific to Trypanosoma brucei gambiense. Mol. Biochem. Parasitol. 113, 127138.

Bosma, G.C., Custer, R.P., Bosma, M.J., 1983. A severe combined immunodeficiency mutation in the mouse. Nature $301,527-$ 530.

Brun, R., Schumacher, R., Schmid, C., Kunz, C., Burri, C., 2001. The phenomenon of treatment failure in human African trypanosomiasis. Trop. Med. Int. Health 6, 906-914.

Burri, C., Keiser, J., 2001. Pharmacokinetic investigations in patients from northern Angola refractory to melarsoprol treatment. Trop. Med. Int. Health 6, 412-420.

Dukes, P., Kaukas, A., Hudson, K.M., Asonganyi, T., Gachumba, J.K., 1989. A new method for isolating Trypanosoma brucei gambiense from sleeping sickness patients. Trans. R. Soc. Trop. Med. Hyg. 83, 636-639.

Gibson, W., Mehlitz, D., Lanham, S.M., Godfrey, D.G., 1978. The identification of Trypanosoma brucei gambiense in Liberian pigs and dogs by isoenzymes and by resistance to human plasma. Tropenmed. Parasitol. 29, 335-345.

Herbert, W.J., Lumsden, W.H.R., 1976. Trypanosoma brucei: a rapid 'matching' method for estimating the host's parasitaemia. Exp. Parasitol. 40, 427-431.

Inoue, N., Narumi, D., Mbati, P.A., Hirumi, K., Situakibanza, N.T.H., Hirumi, H., 1998. Susceptibility of severe combined immunodeficient (SCID) mice to Trypanosoma brucei gambiense and $T$. b. rhodesiense. Trop. Med. Int. Health 3, 408-413.

Lanham, S.M., Godfrey, D.G., 1970. Isolation of salivarian trypanosomes from man and other mammals using DEAE-cellulose. Exp. Parasitol. 28, 521-534.
Legros, D., Fournier, C., Gastellu Etchegorry, M., Maiso, F., Szumilin, E., 1999. Therapeutic failure of melarsoprol among patients treated for late stage $T$. b. gambiense human African trypanosomiasis in Uganda. Bull. Soc. Pathol. Exot. 92, 171-172 [in French].

Magnus, E., Vervoot, T., Van Meirvenne, N., 1978. A card agglutination test with stained trypanosomes (CATT) for the serological diagnosis of T. b. gambiense trypanosomiasis. Ann. Soc. Belg. Med. Trop. 58, 169-176.

Maina, N.W., Kunz, C., Brun, R., 2006. Cryopreservation of Trypanosoma brucei gambiense in a commercial cryomedium developed for bull semen. Acta Trop. 98, 207-211.

Matovu, E., Geiser, F., Schneider, V., Maser, P., Enyaru, J.C.K., Kaminsky, R., Gallati, S., Seebeck, T., 2001a. Genetic variants of the TbAT1 adenosine transporter from African trypanosomes in relapse infections following melarsoprol therapy. Mol. Biochem. Parasitol. 117, 73-81.

Matovu, E., Enyaru, J.C.K., Legros, D., Schmid, C., Seebeck, T., Kaminsky, R., 2001b. Melarsoprol refractory T. b. gambiense isolates from Omugo, north-western Uganda. Trop. Med. Int. Health 6, 407-411.

Médicins Sans Frontières, 2002. Sudan: improving treatments of sleeping sickness and malaria amid civil war. http: / /www.msf.org/ [accessed 20 November 2006].

Mehlitz, D., 1978. Investigations on the susceptibility of Mastomys natalensis to Trypanosoma (Trypanozoon) brucei gambiense. Tropenmed. Parasitol. 29, 101-107 [in German].

Moore, A., Richer, M., 2001. Re-emergence of epidemic sleeping sickness in Southern Sudan. Trop. Med. Int. Health 6, 342-347.

Radwanska, M., Chamekh, M., Vanhamme, L., Claes, F., Magez, S., Magnus, E., de Baetselier, P., Buscher, P., Pays, E., 2002a. The serum resistance-associated gene as a diagnostic tool for the detection of Trypanosoma brucei rhodesiense. Am. J. Trop. Med. Hyg. 67, 684-690.

Radwanska, M., Claes, F., Magez, S., Magnus, E., Perez-Morga, D., Pays, E., Buscher, P., 2002b. Novel primer sequences for polymerase chain reaction-based detection of Trypanosoma brucei gambiense. Am. J. Trop. Med. Hyg. 67, 289-295.

WHO, 1998. African Trypanosomiasis: control and surveillance. Report of a WHO Expert Committee. World Health Organization, Geneva, Technical Report Series No. 881.

WHO, 2004. World Health Report 2004, statistical annex. World Health Organization, Geneva.

Zillmann, U., Mehlitz, D., 1979. The natural occurrence of Trypanozoon in domestic chicken in the Ivory Coast. Tropenmed. Parasitol. 30, 244-248. 